\title{
A Study on the Structure of a Viral RNA
}

\author{
By P. ZipPER AND W. FolKhaRd, \\ Institute for Physical Chemistry, University of Graz, Austria \\ and J. Clauwaert \\ Department for Cell Biology, University of Antwerp, Belgium
}

SAXS was applied to the RNA from bacteriophage $M S 2$. From measurements in a $0 \cdot 1 \mathrm{M} \mathrm{NaCl}$ solution containing $0.5 \mathrm{mM} \mathrm{MgCl}$ the molecular weight of the RNA was obtained as $1.09 \times 10^{6}$ and the radius of gyration as $176 \pm 5 \AA$. The largest diameter of the RNA particle was determined as $620 \AA$. Two cross-section factors were derived from the scattering curve. The one at small angles corresponded to a cross-section radius of gyration $R_{c}=84.3 \AA$ and to a mass per unit length $M_{c}=1890 \AA^{-1}$. From the second cross-section factor, which was found at larger angles, another $R_{c}$ of $9 \cdot 1 \AA$ and another $M_{c}$ of $169 \AA^{-1}$ were derived. The latter value is smaller than the theoretical mass per unit length for a RNA double helix, but it is definitely larger than the value of $91 \AA^{-1}$ found for $M S 2$ RNA in $4 \cdot 6 \%$ formaldehyde solution. It appears to be consistent with a double-helix content of about $70 \%$. Comparison of the scattering curve for $M S 2$ RNA with theoretical curves for various models led to the conclusion that the native $M S 2$ RNA is a flat particle, perhaps an elliptic cylinder with axes of $618 \AA$ and $312-331 \AA$ and a height of 55-110 $\AA$. Further experiments with the RNA were performed in $4.6 \%$ formaldehyde solution at $60^{\circ} \mathrm{C}$. At this temperature the RNA obviously behaves like a random coil, because, besides a cross-section radius of gyration of $7 \AA$ and a mass per unit length of $71 \AA^{-1}$, a persistence length of $59 \AA$ could also be found.

J. Appl. Cryst. (1974). 7, 168

\section{Small-Angle X-ray Scattering Study on the Recognition of a tRNA Substrate by its tRNA Ligase}

\author{
By R. ÖSTERBERG, L. RYMO AND U. LAGERKVIST \\ Department of Medical Biochemistry, University of Göteborg, S-400 33 Göteborg 33, Sweden
}

As the first step of a small-angle X-ray scattering study on the interaction of ligases with their specific tRNA substrate, the lysine-tRNA ligase from yeast has been characterized. Small-angle X-ray data yield a radius of gyration equal to $37.5 \AA$, a molecular weight of 114000 and a volume of $295000 \AA^{3}$. A comparison of the experimental data with the theoretical scattering curves indicates that the shape of the molecule can be represented as an oblate ellipsoid. In the next step solutions containing both ligase and specific tRNA have been studied; the X-ray intensities at small angles increased dramatically compared to the intensities obtained by adding those recorded from separate solutions of ligase and tRNA. However, solutions of ligase and unspecific $t$ RNA did not yield any measurable increases in the intensities. This increase in intensity, $\Delta I$, produced by the specific tRNA, is only a function of the complexes formed between the ligase (L) and its tRNA substrate (S), provided that the intensity constitutes the sum of the intensities for all the particles in the solution. Such $\Delta I$ values have been recorded for two sets of solutions where, in each set, the solutions contained varying tRNA concentrations $(A)$, but a constant ligase concentration $(B)$. When normalized data, $(\Delta I) / B$ against $A$ at constant $B$, for a certain low angle were compared to a series of curves simulated for different complexes of the type $\mathrm{L}_{p} \mathrm{~S}_{q}$, it was found that the curves produced by the $\mathrm{L}_{2} \mathrm{~S}$ complex yielded the best fit. A further support for the formation of such a complex was indicated through the determination of molecular weight and volume. 